\title{
Measuring system and method of determining the Adaptive Force
}

\author{
Laura Schaefer, Marko Hoff, Frank Bittmann \\ Section Regulative Physiology and Prevention, Department of Sport and Health Sciences, \\ University of Potsdam, Brandenburg, Germany
}

This article is distributed under the terms of the Creative Commons Attribution Noncommercial License (CC BY-NC 4.0) which permits any noncommercial use, distribution, and reproduction in any medium, provided the original author(s) and source are credited.

\begin{abstract}
The term Adaptive Force (AF) describes the capability of adaptation of the nerve-musclesystem to externally applied forces during isometric and eccentric muscle action. This ability plays an important role in real life motions as well as in sports. The focus of this paper is on the specific measurement method of this neuromuscular action, which can be seen as innovative. A measuring system based on the use of compressed air was constructed and evaluated for this neuromuscular function. It depends on the physical conditions of the subject, at which force level it deviates from the quasi isometric position and merges into eccentric muscle action. The device enables - in contrast to the isokinetic systems - a measure of strength without forced motion. Evaluation of the scientific quality criteria of the devices was done by measurements regarding the intra- and interrater-, the test-retest-reliability and fatiguing measurements. Comparisons of the pneumatic device with a dynamometer were also done. Looking at the mechanical evaluation, the results show a high level of consistency ( $r^{2}=0.94$ to 0.96$)$. The parallel test reliability delivers a very high and significant correlation $(\rho=0.976 ; p=0.000)$. Including the biological system, the concordance of three different raters is very high $(\mathrm{p}=0.001$, Cronbachs alpha $\alpha=0.987$ ). The test retest with 4 subjects over five weeks speaks for the reliability of the device in showing no statistically significant differences. These evaluations indicate that the scientific evaluation criteria are fulfilled. The specific feature of this system is that an isometric position can be maintained while the externally impacting force rises. Moreover, the device can capture concentric, static and eccentric strength values. Fields of application are performance diagnostics in sports and medicine.
\end{abstract}

Key Words: Adaptive Force, isometric eccentric force, muscle action, motor control, strength measurement system.

Eur J Transl Myol 27 (3): 152-159

In everyday movements one constantly has to adapt adequately to external forces or to the impact of weight and inertia, respectively. Especially the ability to hold a stable resistance against varying external forces was not described earlier as a stand-alone capability. This is why we recently postulated and discussed the concept of Adaptive Force (AF), which shall describe this function. ${ }^{1}$ The AF can be differentiated into three forms of muscle action, which all are captured in one measuring process: 1 . the submaximal isometric AF (AFiso); 2. the maximum isometric $\mathrm{AF}\left(\mathrm{AFiso}_{\mathrm{max}}\right)$; and 3. the maximum eccentric $\mathrm{AF}\left(\mathrm{AFecc}_{\max }\right)$. We are not aware of a technical device which is able to measure these parameters. Therefore, we developed a system with the following special features: It applies increasing forces on the musculoskeletal system without inducing a forced movement (non-desmodromic). A motion does not appear before the individual maximum holding isometric force is exceeded and the subject begins to yield. These demands are met by use of pneumatics that allows to apply time-related force patterns. Due to air compressibility, no desmodromic is forced. Information on the forces between system and subject are indirectly drawn by the data of the pressure sensors. The purpose of this paper is to evaluate the scientific eligibility of this method of measuring the AF.

\section{Material and Methods}

Measuring System

Figure 1 shows the setup of the measuring system. The core piece of the system is the measuring chair, at which the pneumatically driven cylinder including the piston pushrod is fixed. The chair can be adjusted in all three dimensions for an optimal measuring position. The subject is fixed with a seatbelt around the hip. The pushrod in the cylinder can be adjusted reproducibly by 


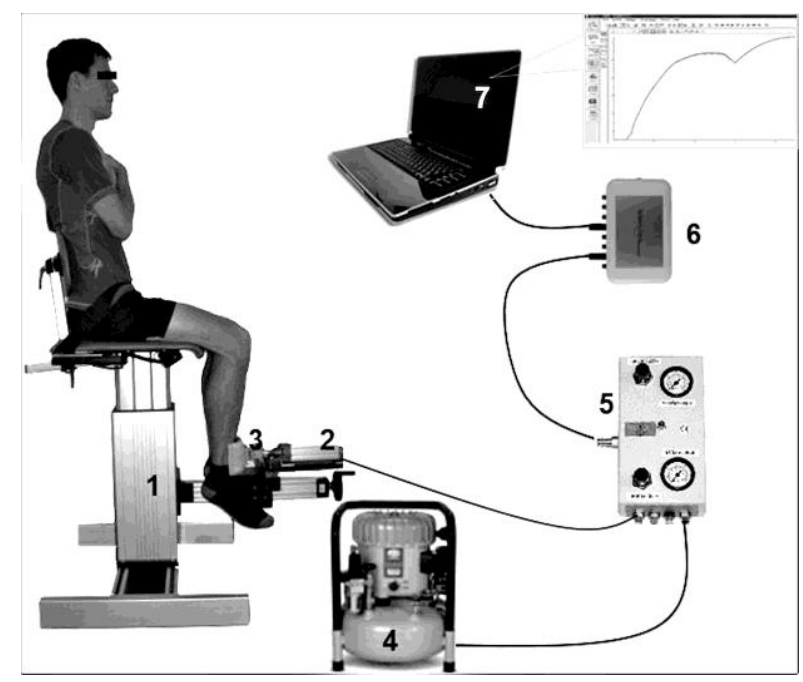

Fig. 1 Measuring system to determine the Adaptive Force

The measuring system consists of a chair (1) including a cylinder with the push rod (2) and the interface (3). The test person takes a standard position on the chair. A compressor with storage tank (4) serves to operate the pneumatic cylinder, which is controlled by a control unit (5). The pressure signal is led through the A-D-converter (6) to the measuring laptop (7). Using the Software DIAdem 10.2 (NI) the data can be saved and processed.

electro-pneumatic means. Time and course of pressure increase are controlled by a control unit with sensor technology (pressure transducer: A-10). The pressure signal is transferred to the A/D-converter (National Instruments (NI), 14-bit, modified by Biovision) and is led to the measuring laptop. There it is recorded and saved by the Software DIAdem 10.2 (NI) and can be further processed. The compressor (Jun-Air, Agre

Table 1. Technical data of the measuring system.

\begin{tabular}{|c|c|}
\hline & Technical specifications \\
\hline pressure & $\begin{array}{l}\text { - system pressure max. } 8 \text { bar } \\
\text { - adjusted pressure: } \\
\text { - slow increase: } 7 \text { bar } \\
\text { - impulsive increase: } 6 \text { bar }\end{array}$ \\
\hline cylinder & $\begin{array}{l}\cdot \text { piston } \varnothing 40 \mathrm{~mm} \\
\text { - up to ca. } 1005 \mathrm{~N}\end{array}$ \\
\hline rise time & $\begin{array}{l}\cdot 1 / 10 \mathrm{~s}-60 \mathrm{~s} \\
\cdot \text { continuous }\end{array}$ \\
\hline stroke way & $\begin{array}{l}\cdot 1-100 \mathrm{~mm} \\
\text { - adjustable }\end{array}$ \\
\hline
\end{tabular}

Kompressor Hobby Star 200; max. 8 bar) generates and stores the compressed air, which is needed for the measurements. Technical data are displayed in Table 1.

\section{Measuring Procedure}

The subject sits on the measuring chair with $90^{\circ}$ flexion in the hip- and knee-joint. The force is transferred through the pushrod and the interface at the distal third of the ventral tibia. It acts in direction of knee flexion, thus into eccentric action of the quadriceps femoris muscle. A standardized starting position of the pushrod is guaranteed by an electro-pneumatic adjustment. During testing, the forearms are crossed in front of the chest. The task for the subject is to adjust a standardized position of the pushrod with a defined starting pressure. For this propose a real-time feedback is shown on a monitor, which displays the pressure-level. The subject has to push against the pushrod to achieve the given (low) pressure-level. During the subsequent increase of the pressure in the system, the adjusted knee angle shall be maintained as long as possible, although the knee would be free to flex (no desmodromic). As soon as the constantly increasing pressure of the system exceeds the maximum isometric strength of the subject (AFiso ${ }_{\max }$ ), the leg will merge into eccentric motion. During this yielding the subject continues to resist as far as possible the cylinders force. The measuring system enables to perform different modifications concerning the forcetime-course. The velocity of the pressure increase and the pressure levels may be varied. Thereby, several measurement settings and the collection of different force forms - also of submaximal forces - are possible. Worth particular mention is the fact that during the submaximal phase of the measurement the subject is not forced to move. It has the possibility to maintain the isometric measuring position if it is able to apply the required force to compensate the applied external increasing load. Moreover, the subject influences the velocity of yielding during the eccentric phase depending on its strength. Nevertheless, the measuring procedure and the device induced force development are standardized. The pressure rising against a stable resistance is recorded prior to the measurement series. The starting force and the slew rate are determined and kept for the measurements of each subject. The detected curve serves as the reference for evaluation of the AF.

\section{Force-time-curve of Adaptive Force}

Figure 2 shows the typical course of the air pressure during measurements of a test person (lower curve) and against a stable resistance (reference curve, upper curve). The reference curve displays the pressure increase without deviation against a completely stable resistance. Looking at the curve of the subject, one can see several deviations compared to the reference during the pressure increase. This is due to slight divergences of the isometric position of the subject during adaptation to the increasing pressure of the device. When the 


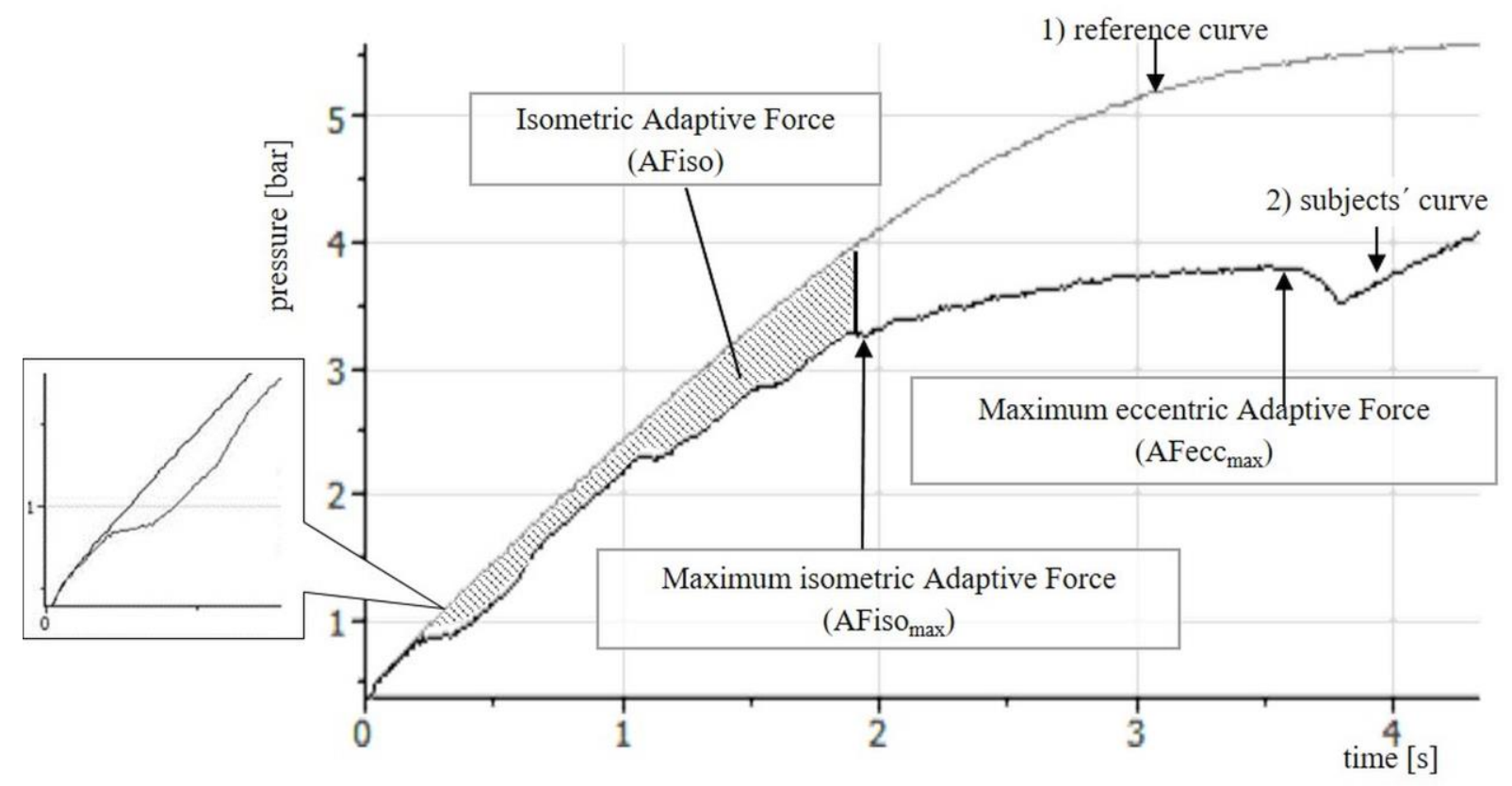

Fig 2. Course of pressure of a measurement curve of a subject (2) compared to a reference curve (1) against a stable resistance; both measured with the AF-system. The marked area illustrates the AFiso. (Reproduced from the Open Access article by Hoff M, et al. Eur J Transl Myol 2015; 10.4081/ejtm.2015.5183) ${ }^{1}$

subject deviates from the isometric position, the pressure curve will increase either slower or faster compared to the reference curve. When the subject yields (quadriceps femoris muscle merges into eccentric), the pushrod drives out and the pressure curve increases slightly slower because in the cylinder a higher spatial volume is available. This condition remains as long as the subjects yield. As soon as it is able to maintain a stable position again, the curve will increase faster, i.e., parallel to the reference curve. If the subject pushes the pushrod into the cylinder (concentric action of quadriceps femoris muscle), the curve increases temporarily faster and then the curve get closer to the reference again. Especially in the first $2 / 3$ of the pressure rise, the subjects' curve runs in several phases nearly parallel to the reference. This stable isometric - phases are getting shorter with increasing pressure. Finally, the subject cannot maintain the static position anymore. It passes into eccentric action. But also during this yield, the pressure and with this also the force increases further. After exceeding the maximum eccentric force $\left(\mathrm{AFecc}_{\max }\right)$ of the test person, the pressure drops and the subject continuous to yield (in Fig. 2 at $3.7 \mathrm{~s}$ and 3.8 bar). The pushrod moves completely out of the cylinder until it reaches its endstop (here at $3.8 \mathrm{~s}$ and $3.5 \mathrm{bar}$ ). Thereby conditions of stable resistance result. Hence, a further pressure increase occurs. Since this is generated in the system, it is irrelevant for the subjects force evaluation. The submaximal isometric Adaptive Force (AFiso) of the test person is represented graphically by the difference of the subjects curve to the reference. The closer the subjects curve runs to the reference, the better the AF. The measuring system allows to determine different qualities of the AF. ${ }^{1}$ The measurement of the maximum isometric force $\left(\right.$ Fiso $\left._{\max }\right)$ by pushing against the complete fixed pushrod of the device is also possible. Furthermore, an unconventional measurement is feasible by pushing concentrically against the pushrod.

Additional - unconventional - measuring mode: the maximum concentric-isometric force (Fcon-iso ${ }_{\max }$ )

Additionally to the above described modes whereby the subject reacts, the maximum concentric-isometric force (Fcon-iso ${ }_{\max }$ ) can also be recorded with the pneumatic system. If the subject pushes the pushrod into the cylinder, the air will be compressed in the closed passive pneumatic system. Therefore, the resistance and thus the reaction force between subject and system gets constantly higher. The determined curve (Fig. 3 bottom) represents the pressure course generated by the subject and by this, the force over time. At the beginning the muscle acts concentrically. With increasing resistance, the concentric motion becomes slower until it ends in a standstill. The concentric-isometric maximum is reached: the Fcon-iso max $_{\text {. }}$

\section{List of evaluation measurements}

Prior to the measurements with subjects, the system was investigated concerning its mechanics during measurements against a stable resistance. $\mathrm{N}=10$ trials for test-retest-reliability as well as parallel-test 

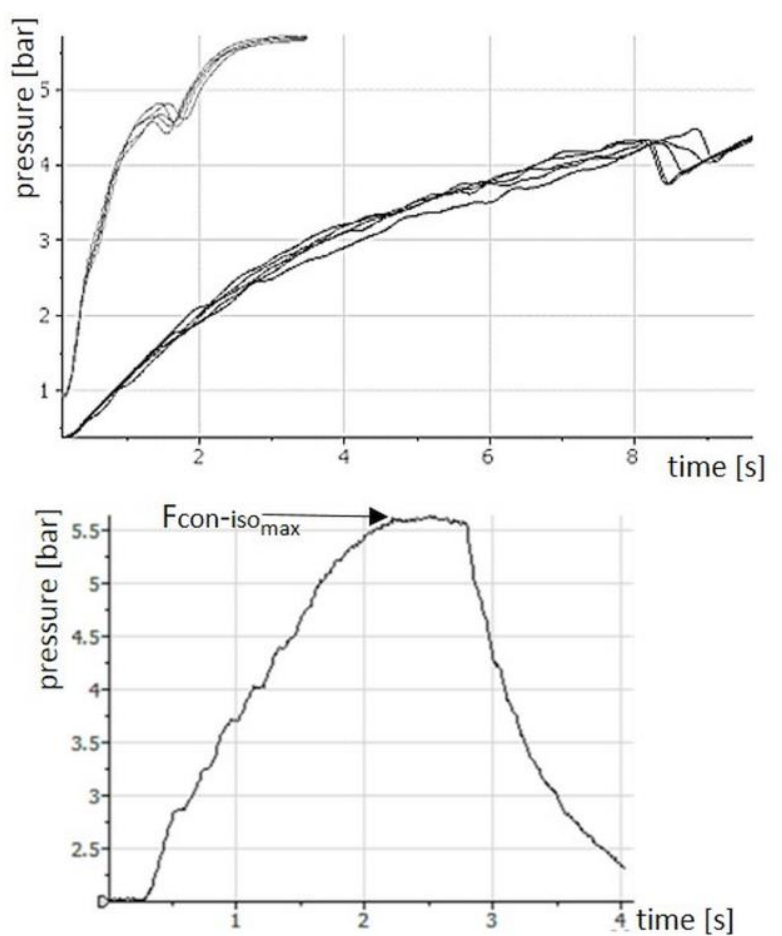

Fig. 3. Pressure curves [bar] measured by the system SeBit knee. Above: Five trials of measuring the AF of one subject during slow (black) and rapid (grey) pressure increase. The data are filtered with butterworth filter (filter degree 10, cut-off frequency $3 \mathrm{~Hz}$ ). Bottom: One trial of maximum concentric-isometric force Fcon-iso $\mathrm{Sax}_{\max }$.

measurements were done using additionally a strain gauge to verify the reliability and the validity of the system. To check the reliability of the mechanical system in interaction with subjects, the following measurements were done: 1) Interrater-reliability: $n=3$ subjects were measured 5 times in a row by three different testers; resting period: 60s. 2) Longtime testretest-reliability: $n=4$ subjects were measured bilaterally once a week over five weeks at the same day and time (one subject only performed the trials over three weeks); the following trials were performed randomized at each measuring session: 5 times normal pressure increase with a pre-pressure of 0.4 bar (slow; $\mathrm{AFecc}_{\max }$ reached within approx. $4 \mathrm{~s}$ ); 5 times impulsive pressure increase with a pre-pressure of 1 bar (rapid; $\mathrm{AFecc}_{\max }$ reached within approx. $1.5 \mathrm{~s}$ ); 3 trials of the concentric-isometric force. Resting period: 60 s. 3). Short-time test-retestreliability: $n=10$ subjects were measured 50 times in a row (resting period: $60 \mathrm{~s}$ ) either with the slow or the rapid increase: $n=4$ solely with slow increase, $n=6$ with rapid and slow increase on two different measuring days with one week in-between. This should provide indications of fatiguing effects as a result of repeated measurements.

\section{Participants}

In total $\mathrm{n}=15$ (m: 7 ; f: 8) healthy subjects participated voluntarily in the study. The subjects were aged between 22 and 39 years $(27.4 \pm 5.5)$, weighed average $68.3 \mathrm{~kg}( \pm 9.2)$ and were $173.23 \mathrm{~cm}( \pm 8.2)$ tall. The exclusion criteria were knee total endoprosthesis and knee complaints. Everyone completed a questionnaire concerning personal and medical information. The study was conducted according to the declaration of Helsinki and local ethical permission was given. All subjects were informed in detail and gave their consent to participate

\section{Data processing}

For all measurements the Software DIAdem 10.2 (NI) was used. The sampling rate was set at $100 \mathrm{~Hz}$. The software is further used for data preparation. The statistical analyses were done with IBM SPSS Statistics. The Shapiro-Wilk test was performed to check normal distribution. If the data were normal distributed, parametric procedures were chosen, if not non parametric procedures were used. The significance level was set at $\alpha=.05$. Looking at the mechanics of the system (measuring against a stable resistance), the testretest-reliability $(n=10)$ was evaluated by splitting each curve into ten intervals of 100 measuring points. The arithmetic mean values were calculated of each interval. The mean values were compared with the Levene's test for homogeneity of variances. For the parallel-testreliability, the pressure curve was compared to the curve of a strain gauge by using the Spearman's rank correlation coefficient. Measuring the AF of a subject, the maximum pressure value of each measuring curve was determined, which represents the maximum eccentric Adaptive Force $\left(\mathrm{AFecc}_{\max }\right)$. This is used to calculate the torque in Excel by $\mathrm{M}=\mathrm{P}^{*} \mathrm{~A} * \mathrm{r}$, with $\mathrm{A}=12.57 \mathrm{~cm}^{2}$ (cylindrical surface) and $\mathrm{r}$ as the individual radius of each subject (distance between the middle of the condyles of femur and the middle of interface). The arithmetic mean of the $\mathrm{AFecc}_{\max }$ values of each trial, subject and series of measuring (interrater/long-time test-retest-reliability) were calculated and transferred to SPSS. Concerning the short-timereliability, the 50 trials were separated in five intervals with each ten trials (trial 1-10, 11-20, 21-30, 31-40, 4150). The ten $\mathrm{AFecc}_{\max }$ values of each interval were averaged. Subsequent, the Friedman two-way analysis of variance by ranks was used to determine potential differences between the intervals. For the long-timereliability the univariate Analysis of Variance (ANOVA) for repeated measurements was performed. For the interrater-reliability the Intraclass-CorrelationCoefficient $\operatorname{ICC}(3,3)$. The coefficient of variation $(\mathrm{CV})$ was calculated for several considerations. 

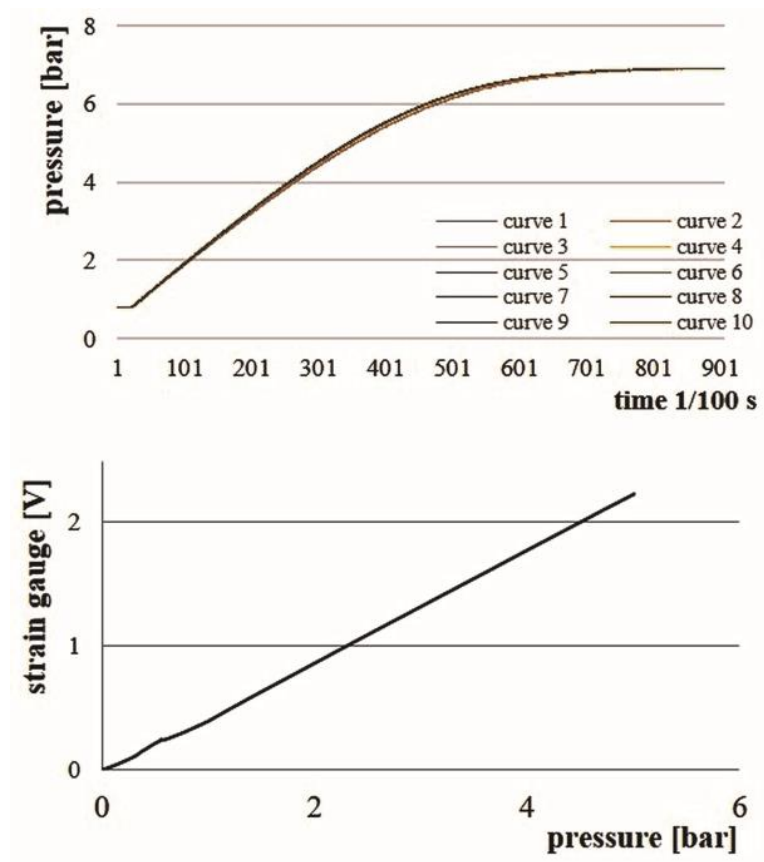

Fig 4. Above: Diagram of ten pressure curves measured in a row (sampling rate $100 \mathrm{~Hz}$ ). Below: Scatter diagram between rises of the strain gauge and of the pressure signal.

\section{Results}

\section{Evaluation of the measuring system}

The measurements against a stable resistance reflect a high level of consistency: The evaluation of the mean values of different curve intervals during ten repeated measurements (Fig.4 above) delivers in the Levene'stest for homogeneity of variances between the ten trials $\mathrm{F}(9,90)=0.004, \mathrm{p}=1.0$. Also the comparison of the system with a strain gauge (parallel-test-reliability) shows a very high and significant correlation of $\rho=0.976$ $(p=0.000) \quad$ (Fig.4 below). In connection with the biological system the statistical reliability reduces slightly. Nevertheless, the interrater-test concerning $\mathrm{AFecc}_{\text {max }}$ with $\mathrm{n}=3$ subjects shows a high concordance of three different raters (ICC $[3,3]=0.974, p=0.001$, Cronbachs-alpha $\alpha=0.987)$. The test-retest with $n=3$ subjects over five weeks speaks for reliability of the device. The measuring shows no statistically significant changes of the force values calculated out of the mean pressure data of right and left side of each subject (AFecc: $\left.\quad F(4,8)=1.510, \quad p=0.287, \quad \eta^{2}=0.430\right) ; \quad$ AFecc rapid: $\mathrm{F}(4,8)=1.551, \mathrm{p}=0.0276, \eta^{2}=0.437$; MVC static: $\left.\mathrm{F}(4,8)=0.413, \mathrm{p}=0.795, \eta^{2}=0.171\right)$.

The validity was confirmed by the parallel-test (see above).

\section{Behavior of $A F e c c_{\max }$ during frequently repeated measurements}

The Friedman test of the values of $\mathrm{AFecc}_{\max }$ results in no significant difference between the intervals for the rapid pressure increase $\left(\chi^{2}(4)=7.333, p=0.119, n=6\right)$. The Friedman test of the $\mathrm{AFecc}_{\max }$ data with slow pressure increase delivers a significant result $\left(\chi^{2}(4)=21.04, p=0.000, n=10\right)$. The post-hoc test (DunnBonferroni) shows, that especially the first interval (I1) differs significantly from the others (I1 vs. I3: $\mathrm{z}=2.0$, $\mathrm{p}_{\text {adjust }}=0.047, \mathrm{r}=0.63 ; \mathrm{I} 1$ vs. I4: $\mathrm{z}=2.2, \mathrm{p}_{\text {adjust }}=0.019$, $\mathrm{r}=0.696$; I1 vs. I5: $\mathrm{z}=2.9, \mathrm{p}_{\text {adjust }}=0.00, \mathrm{r}=0.92$; I2 vs. I5: $\mathrm{z}=2.0, \mathrm{p}_{\text {adjust }}=0.047, \mathrm{r}=.63$ ). This may be explained by five subjects, which show significant higher torque values within the first interval compared to the subsequent 40 measurements (Fig. 5). All of these athletes stated that they are mainly performing explosive power sports. The other subjects were primarily performing endurance sports.

The dispersion of five trials in a row of one subject
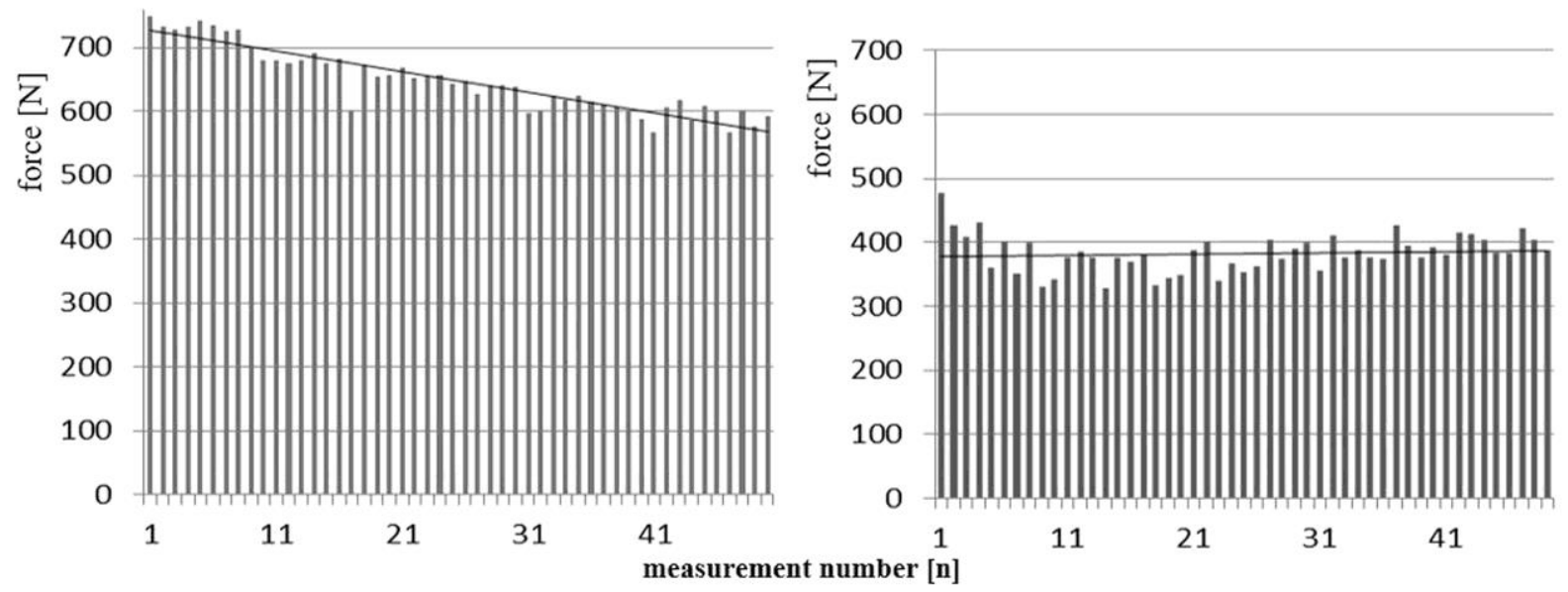

Fig 5. Values of $\mathrm{AFecc}_{\max }$ of two subjects during 50 repetitions with a linear trend line. (left: R2=0.868; right: $\mathrm{R} 2=0.012$.$) .$ 


\section{Measuring the Adaptive Force \\ Eur J Transl Myol 27 (3): 152-159}

during fast and slow increase is displayed in Fig. 3 above. The $\mathrm{CV}$ of 50 repeated measurements amounts $5.4 \%( \pm 1.8)$ with slow pressure increase $(n=10)$ and $2.9 \%( \pm 1.5)$ with rapid pressure increase $(n=6)$.

\section{Discussion}

The results of the present data underpin the assumption that the AF-measuring system meets the quality criteria of scientific research, but because of the small sample size this has to be confirmed by further measurements. For the quantification of the AFiso (Fig. 2, marked area) the authors propose to use the deviation of the subjects curve from the reference. Thereby, the area between the curves from the starting point to the $\mathrm{AFiso}_{\max }$ would be utilized. The maximum isometric AF is marked by the highest point of the last isometric period (defined by the last isometric interval $>0.25 \mathrm{~s}$ ). The submaximal $\mathrm{AF}$ could be quantified by the difference of both curves: the smaller the area between the graphs, the better the submaximal isometric AF. In addition, the measurement provides the $\mathrm{AFisO}_{\text {max }}$ at the transition of isometric to eccentric muscle action. We consider it an innovative approach because the maximum isometric strength was so far measured by actively pushing against a rigid passive sensor. The latter could be understood also as a kind of forced control by the device, since static conditions are inevitably given. The presented device provides the transition threshold of isometric to eccentric activity, thus, the optimal isometric holding ability. The relation between it and the conventional maximum isometric strength is not yet clear. However, we assume that pushing against a stable resistance (like isometric strength is usually measured) seems to involve different control strategies of the neuromuscular system than holding a force, that is impacting from external, while maintaining an isometric position (as performed with the presented system). ${ }^{2}$ This hypothesis is derived from the findings of Enoka (1996) and Duchateau \& Baudry (2013). They draw the conclusion, that eccentric muscle action is based on a more complex neuronal motor control strategies. 3,4

We hypothesize moreover a difference between pushing and holding isometric muscle action, ${ }^{5-8}$ but comparative investigations remain an issue for future research. Anyhow, with the presented measurements the maximum eccentric Adaptive Force $\mathrm{AFecc}_{\max }$ could be assessed. It refers to the maximum strength within the given setting. The relation between this and otherwise measured eccentric strength (e.g. isokinetic) is also not yet clear. In order to identify possible determinants, we made an investigation to examine the influence of the pre-activation (starting power) and the slew rate of pressure on the $\mathrm{AFecc}_{\max } \cdot{ }^{9}$ Thereby, we pursued whether the $\mathrm{AFecc}_{\max }$ will further increase under optimal conditions of the pre-activation (manuscript in preparation) so that it approaches the absolute strength postulated by Schmidtbleicher. ${ }^{10,11}$ The measuring system can also be used to measure the maximum isometric strength in the conventional manner (Fiso ${ }_{\max }$ ) when the subject itself applies force on the fixed pushrod. Furthermore with the presented device the strength of a concentric-isometric muscle action is determinable (Fcon-iso max $_{\max }$ ). We suppose, that this is similar to the Fiso $_{\max }$, but further investigations remain to be performed. In summary, one can assess several strength abilities with the measurement system: maximum and submaximal isometric AF: $\mathrm{AFiso} \mathrm{AFiso}_{\max }$; maximum eccentric $\mathrm{AF}$ (slow/rapid): $\mathrm{AFecc}_{\max } / \mathrm{rapAFecc}_{\max }$; maximum isometric force: Fiso $_{\max }$; maximum concentric-isometric force: Fconiso $_{\max }$

\section{Reproducibility of repeated measurements}

The device-specific reliability is very high. Also compared to other measuring methods the reproducibility of the measurement with subjects is good as shown by pilot study comparing isokinetic, AFsystem and static systems (manuscript in preparation). The higher variability may reflect mostly the biological variability. Concerning a faster force increase, the variation decreases. To sum up, the presented measuring system displays a satisfying low variation and therefore a sufficient reproducibility during repeated measurements concerning the $\mathrm{AFecc}_{\max }$.

Behavior of AFecc $_{\max }$ during frequently repeated measurements

Another factor concerning repeated measurements could be fatigue. The measured isometric-eccentric activity seems to be rarely susceptible to fatigue under the applied conditions. This is suggested by the fact, that for all subjects no significant strength loss appears during the rapid force increase. On the other hand, during the slow force increase, specifically the first 10 measurements deviates significantly from the last 40 trials of $\mathrm{AFecc}_{\max }$. The significant decrease within the first measurements in some subjects still needs to be clarified. The fact that those subjects come from explosive power sports could suggest that different muscle fiber types could be concerned. The significant higher strength values within the first ten measurements could be explained by a greater proportion of fast twitch muscle fibers. Habituation effects drop out, since a force decrease is present. Again, further investigations are needed.

\section{Methodological critique}

A point of criticism is the stick-slip-effect of the pneumatic device. The pushrod is not frictionless, that is why the pressure curve shows the jerky movements as can be seen in Fig. 2. Potentially, this could influence the maintaining the isometric phase. Furthermore, the applied pressure increase was chosen identical for each subject. More favorable would be an individualized rise adjusted to the isometric MVC of each subject. Additionally, a recording of the angle of the knee joint would be helpful to determine the moment in which the 


\section{Measuring the Adaptive Force \\ Eur J Transl Myol 27 (3): 152-159}

leg starts to merge into eccentric muscle action. With regard to the measurement protocol one has to conclude from the frequently repeated measurements, that more than ten measurements in a row might be problematic.

\section{Characteristics of the pneumatic device}

The presented measuring system allows to evaluate instantaneous adaptability of the sensorimotor system to external impacting varying forces under quasi-isometric conditions, inter alia with submaximal intensities (AFiso). To our knowledge, this is realized by no other device until now. In real life motions, which normally take place with submaximal intensities, no forced control through external impact takes place. It is up to the human organism in what way it reacts to the dynamically changing external forces. We have shown, how such a measurement could function in principle. At the present stage, the system is able to realize different slew rates of force. Future versions could simulate nearly any time-force-courses. Further work must show which of them is best for capturing the submaximal sensorimotor adaptability. To sum up, the special features of the AF-system are firstly the possibility to perform strength measurements with submaximal forces. Secondly to maintain a quasiisometric position during the measurement until the impacting external force exceeds the isometric strength of the subject. The authors consider this as a unique and distinguishing feature. In the view of the authors, the presented measurement system to assess the Adaptive Force enables to capture an aspect of neuromuscular functionality, which was not considered so far. The measurement system satisfies the scientific requirements for the $A F e c c_{\text {max }}$ and the $\mathrm{Fiso}_{\text {max }}$ at the present state of data. Further investigation are needed to confirm this outcome. The points of criticism will be eliminated in new measuring series with other devices including a frictionless cylinder. This second generation system may provide better conditions to evaluate the AFiso $_{\text {max }}$. Meantime, systems for the extensors and flexor muscles of arm and leg were constructed. The apparatus for the arm extensor is already in evaluation phase. The potentials of this approach are not yet established. Fields of application could be open up in diagnostics of physical performance in sports and medicine. It would also be conceivable to include the system in training of competitive athletes. All this will depend on results of further basic research. Anyhow, in comparison to isokinetic systems compressed air system enables to determine different strength types with little effort, easy use and low cost, specifically for eccentric strength that is hard to be consistently assessed.

\section{List of acronyms}

AF - Adaptive Force

$\mathrm{AFecc}_{\max }$ - maximum eccentric AF

AFiso - submaximal isometric AF

$\mathrm{AFiso}_{\max }-$ maximum isometric $\mathrm{AF}$
CV - Coefficient of variation

Fcon-iso $_{\max }$ - Maximum concentric-isometric force

Fiso $_{\max }$ - maximum isometric force

NI - National Instruments

\section{Author's contributions}

The concept of the study was mainly developed by FB, partly by $\mathrm{MH}$ and LS. All authors have contributed substantially to the conception and the design of the work. The acquisition of test persons and the measuring process was organized and performed by $\mathrm{MH}$ and LS. The analysis of data was mainly done by LS, partly by $\mathrm{MH}$. The interpretation of data was done by all authors. The draft of the work was done partly by $\mathrm{MH}$ and mainly by LS. All authors have contributed in the typescript revision process, especially FB. All authors gave their final approval of the version to be published and agreed to be accountable for all aspects of the work.

\section{Acknowledgments}

We thank Prof. em. Dr. Henning Läuter, Emeritus Professor of Mathematical Statistics, Institute of Mathematics, University of Potsdam, for the discussion of the statistical procedure. This project was funded by the German Federal Ministry of Economy and Technology (Project ZIM KF2262301FO9). The employment in 2011 of Marko Hoff and Laura Schaefer was funded by the German Federal Ministry of Economy and Technology (BMWi). The prototype for knee extensor muscles was constructed in cooperation with the company Seifert Drucklufttechnik $\mathrm{GmbH}$ (Bernsbach, Germany). We acknowledge the support of the Deutsche Forschungsgemeinschaft and Open Access Publishing Fund of University of Potsdam.

\section{Conflict of Interest}

The authors state no conflict of interests.

\section{Corresponding Author}

Laura Schaefer, Karl-Liebknecht-Str. 24-25, House 24, R. 1.14, 14476 Potsdam, Germany.

E-mail: 1schaefe@uni-potsdam.de

E-mails of coauthors

Marko Hoff: marko.hoff@gmail.com Frank Bittmann: bittmann@uni-potsdam.de

\section{References}

1. Hoff M, Schaefer L, Heinke N, Bittmann F. Eur J Transl Myol - Basic Appl Myol 2015;25:183-189. doi: 10.4081/ejtm.2015.5183.

2. Schaefer L. Synchronisationsphänomene myotendinöser Oszillationen interagierender neuromuskulärer Systeme - mit Betrachtung einer Hypothese bezüglich unterschiedlicher Qualitäten isometrischer Muskelaktion. Doctoral dissertation, The University of Potsdam, Germany. 2014. Available: http://opus.kobv.de/ubp/volltexte/ 2014/ 7244 


\section{Measuring the Adaptive Force}

Eur J Transl Myol 27 (3): 152-159

3. Duchateau J, Baudry S. Insights into the neural control of eccentric contractions. J Appl Physiol 2013;116:1418-25.

4. Enoka RM. Eccentric contractions require unique activation strategies by the nervous system. J Appl Physiol 1996;81:2339-46.

5. Schaefer L, Bittmann F. Are there two forms of isometric muscle action? Results of the experimental study support a distinction between a holding and a pushing isometric muscle function. BMC Sports Sci Med Rehabil 2017;9:11. doi: 10.1186/s13102-017-0075-z.

6. Hunter SK, Ryan DL, Ortega JD, Enoka RM. Task Differences With the Same Load Torque Alter the Endurance. J Neurophysiol 2002;88:3087-96.

7. Rudroff T, Barry BK, Stone AL, et al. Accessory muscle activity contributes to the variation in time to task failure for different arm postures and loads. J Appl Physiol 2007;102:1000-6.

8. Rudroff T, Justice JN, Holmes MR, et al. Muscle activity and time to task failure differ with load compliance and target force for elbow flexor muscles. J Appl Physiol 2011;110:125-36.

9. Schaefer L, Hoff M, Barnick D, Bittmann F. Einfluss der muskulären Vorspannung auf die maximale schnelle Adaptive Kraft: Der BoostEffekt. Poster presentation at the jubilee congress of the German Society of Sport medicine and prevention (DGSP) in Berlin (3rd poster prize). Deutsche Zeitschrift für Sportmedizin 2012;63:200.

10. Schmidtbleicher D. Strength Training: Structure, Principles and Methodology, 2006. Access on 22th March 2016 under http://www.salisbury.edu/ sportsperformance/Articles/STRENGTH\%20TRI ANING\%20STRUCTURE\%20PRINCIPLES\%20 AND\%20METHODOLOGY\%20\%20SCHMIDTBLEICHER.pdf

11. Bührle M, Schmidtbleicher D, Ressel H. Die spezielle Diagnose der einzelnen Kraftkomponenten im Hochleistungssport. 1983;3:11-6. 